\title{
Is the cerebellum ready for navigation?
}

\author{
Laure Rondi-Reig and Eric Burguière \\ Laboratoire de Physiologie de la Perception et de l’Action, UMR CNRS 7124, \\ 11 place Marcellin Berthelot, Collège de France, 75005 Paris, France \\ tel : 01-44-27-14-30; Fax : 01-44-27-13-82 \\ e-mail : laure.rondi-reig@college-de-france.fr ; eric.burguiere@college-de- \\ france.fr
}

Keywords: cerebellum; navigation; declarative learning; procedural learning; LTD 
The cerebellum has first been considered to contribute to motor control and coordination (Holmes G., 1939). Since then, many other functions have been attributed to the cerebellum, such as motor learning (Thompson and Kim, 1996; Thach, 1998) or cognitive functions (Leiner et al., 1993) including spatial navigation (Lalonde, 1997; Petrosini et al., 1998; Rondi-Reig et al., 2002). However, the role of the cerebellum in cognitive function is still a matter of strong debate. Why is the road to prove the role of the cerebellum in non-motor functions so long and difficult? A recurrent problem is the difficulty in dissociating motor deficits per se from cognitive impairment in paradigms in which the measured variable is behaviour. Is there clear evidence in favour of the involvement of the cerebellum in cognition and more particularly in navigation? By contrast, could all cognitive dysfunctions be explained by a deficit in a common (motordependant?) process for which cerebellar patients or animal models display a deficiency? This review attempts to give a critical, although not exhaustive, overview of different results coming from anatomical data, experimental paradigms and models showing a possible implication of the cerebellum in navigation.

\section{Spatial navigation: a dual-process function}

Spatial navigation is a cognitive function that can be defined as a selfcontrolled movement in space toward a non-visible goal. This function requires the integration of both internal (vestibular, proprioceptive or kinesthetic) and external (visual, olfactive, or auditory) sensory-motor information. Whereas the 
first observations concerning the potential role of the cerebellum in cognitive function were made in humans, the putative role of the cerebellum in navigation essentially comes from experiments with animal models presenting a cerebellar impairment. Traditional paradigms used are mazes in which the animal has to localize the relative position of a non-visible goal and then to orient itself toward this goal as in the classical Morris water maze (Morris et al., 1984) (see Fig. 1). Spatial navigation can therefore be defined as a dual-process function that requires the acquisition of at least two complementary processes: the organization of the spatial representation of the environment (declarative component) and the acquisition of a motor behavior adapted to the specific context (procedural learning). An important question currently under debate concerns the exact role of the cerebellum in these processes.

Figure 1

1.1. Patients with cerebellar lesions show unexpected cognitive deficits

In humans, a review of current literature concerning cerebellar patients shows a very heterogeneous range of propositions for cerebellum functions in cognitive processes. Reports of impairments range from visuo-spatial recall or serial reaction time task (Gomez-Beldarrain et al., 1998; Botez et al., 1989; Drepper et al., 1999) to higher cognitive levels such as judging the timing of events (Ivry, 1997), anticipatory planning (Grafman et al., 1992) or verbal fluency tasks (Hubrich-Ungureanu et al., 2002).

A number of neuropsychological studies have indicated that cerebellar damage affects the processing of spatial information. Friedreich's ataxia and olivo-pontocerebellar atrophy (OPCA) patients, mostly characterized by atrophy of afferent cerebellar fibers or by damaged olivary and pontine nuclei and cerebellum 
respectively, were evaluated with a battery of neuropsychological tests. The Rey complex figure test evaluates visuo-spatial functioning. In this task, patients are either asked to copy a complex figure or to draw the figure from memory (see an example of the drawing in Fig. 2). Different components can be evaluated: the accuracy, the location (quantitative evaluation) and the organization (qualitative evaluation) of the drawings. Cerebellar patients evaluated with the copy version were both quantitatively and qualitatively impaired in this complex task (BotezMarquard and Botez, 1992), suggesting a deficit in visuo-spatial organization.

Figure 2

Neuro-logical and -psychological evaluation of other patients with diseases confined to the cerebellum also revealed that 'cerebellar cognitive affective syndrome' included visual-spatial impairment (Schmahmann and Sherman, 1997) in both versions of the Rey complex figure test (copy and memory), and was reflected in the disorganization of the sequential approach to the drawings and the conceptualization of the figures. The patients were also tested in the Hooper task, which tests the individual's ability to organize visual stimuli. The test consists of multiple line drawings, each showing a common object (such as an apple or a ball) that has been cut into several pieces. The pieces are scattered on the page like parts of a puzzle. The patient's task was to tell what the object would be if the pieces were put back together correctly. Patients were not significantly impaired in this task, suggesting an absence of visual organization deficit per se in cerebellar patients. A difference, however, between these two tasks is the verbal response required in the Hooper task and the motor response in the Rey complex figure test. This distinction might support the alternative explanation that impairment in the Rey test might also come from the motor component of the 
task. An argument in favor of the cognitive hypothesis, however, comes from the fact that the deficit was observed regardless of the severity of the dysmetria (i.e. the lack of coordination of movement), suggesting that the observed impairment could not be explained by difficulties with motor control. Some patients also showed simultagnosia, i.e. typically could see individual objects but were unable to recognize a visual scene as a whole.

Clinical observations of cerebellar patients have given accumulative evidence of impaired performances in a number of cognitive tasks. However, the interpretation of these deficits as a true cognitive impairment remains unclear. Taken together, these clinical observations suggest more than they demonstrate a possible visuo-spatial impairment in cerebellar patients.

1.2. The mutant cerebellar mouse as a first animal model : progress and limitation Cerebellar mutant mouse studies first reported a possible implication of the cerebellum in navigation (see Table 1) (Lalonde, 1997). These mutant mice all performed very poorly in the traditional paradigm of the Morris water maze when required to find a hidden platform from random departure locations (Fig. 1A).

Table 1

However, the significant ataxia in these models complicates the interpretation of a role of the cerebellum in the spatial learning process per se. In order to overcome this problem, different approaches have been used. First, the presence of the ataxic syndrome in cerebellar mutant was one of the reasons for using a water paradigm, as the motor responses required during swimming are less affected by cerebellar damage than those required during walking or running (Fortier et al., 1987). Second, the lack of a correlation between spatial and visuo- 
motor deficits could be achieved by either statistical analysis (Lalonde and Thifault, 1994) and/or the comparison of performance in paradigms allowing navigation toward a hidden or a visible goal (see Fig. 1).

Goodlett and collaborators first reported that Purkinje cell degeneration (pcd) mutant mice were deficient in the hidden goal paradigm but not in the visible paradigm, strengthening the idea of a role for the cerebellum in spatial learning (Goodlett et al., 1992). Gandhi and collaborators confirmed this previous report using OX7, an immunotoxin selective to Purkinje cells, and showed in addition that despite the lack of motor deficit in their model, spatial learning toward a hidden goal was still impaired (Gandhi et al., 2000).

Taken together, these results were the first demonstration of the involvement of the cerebellum, and even more specifically of Purkinje cells, in navigation. It was clear that this deficit was not due to motor impairment. A question however remained unsolved. As mentioned above, navigation is a dual process requiring both the organization of the spatial representation of the environment (declarative component) and the acquisition of a motor behavior adapted to the specific context (procedural learning). Is the cerebellum involved in both processes or only in the procedural component? If the cerebellum is involved in the procedural component, does that component also participate in declarative knowledge?

\subsection{Declarative versus procedural learning.}

Investigations into the participation of the cerebellum in navigation raise the question of the characterization of its exact role in spatial tasks. Schenk and Morris defined declarative spatial memory as "the representation of knowledge in a form which describes the position of the escape platform in relation to other 
cues of the environment" whereas procedural learning was defined as "the representation of stimulus-responses necessary to guide the animal to the correct location" (Schenk and Morris, 1985). The studies mentioned above concerning cerebellar patients or cerebellar mouse models did not attempt to distinguish between cognitive versus procedural impairments. In recent years, authors have tried to distinguish exactly which processes computed by the cerebellum are implicated and essential for accurate navigation.

Using hemi-cerebellectomized rats in order to obtain a cerebellar model with partial deficit and no strong basal motor impairment, Petrosini and Molinari's group gave some answers. They examined the role of the cerebellum as an essential structure for the elaboration of a correct representation of the environment or as a player in the procedural component required during navigation. They tried to characterize the exact origin responsible of the poor performances observed in hemi-cerebellectomized rats relative to controls animals during spatial navigation tasks, using various protocols, notably Morris water maze (MWM) and T-maze paradigms (Petrosini et al., 1996; Mandolesi et al., 2001; Molinari et al., 1997). In the MWM task, the authors measured latencies of hemi-cerebellectomized rats and observed the strategies used (direct finding, extended or restricted searching, circling) in order to reach a hidden platform from different departure locations (place phase) (see Fig. 1A). This task was done before or after the hemi-cerebellectomy, and before or after a cue phase in which the platform was visible (see Fig. 1B). Performances were different depending on the protocol used; hemi-cerebellectomized rats displayed a severe impairment if they were trained first in the place phase. However, if rats were trained before this 
place phase with a cue phase or if they were preoperatively trained, their deficit observed in the MWM were significantly reduced (see Fig. 3).

Figure 3

With these results, authors proposed a crucial role of the cerebellum during the acquisition, but not the retention, of a spatial task (Petrosini et al., 1998) (Hilber et al., 1998). In addition, they proposed a predominant role of the cerebellum in the organization of the motor behaviour adopted to reach the target (procedural component) rather than in the elaboration of an internal map of the environment necessary to localize the target (Leggio et al., 1999). However, in a recent study, they proposed that the acquisition of the declarative spatial memory requires learning of a correct explorative behaviour suggesting that the procedural impairment observed in cerebellar animals could then lead to a more cognitive impairment (Mandolesi et al., 2003).

Others studies have tried to dissociate motor and cognitive demands during spatial navigation (Gandhi et al., 2000; Martin et al., 2003). With pharmacological and genetic approaches, authors obtained models with reduced motor skills deficits but still significant impairment during spatial navigation tasks. These studies are in agreement with the proposition of a role for the cerebellum in spatial processing, in synergy with others brain areas (Petrosini et al., 1998). They also suggested that performances during navigation tasks were the consequence of both processes. One is a declarative component which allows learning where the target is and could involve the hippocampus and associated areas. The other is a procedural component necessary to develop an effective exploration behavior and could involve the cerebellum and other associated brain areas. 


\section{The anatomical substrate for the putative role of the cerebellum in navigation}

2.1. The cerebellum receives multi-sensorial inputs

The anatomical connections of the cerebellum represent a good substrate for the putative role of the cerebellum in navigation. In mammals, the cerebellum receives proprioceptive, somato-sensory, visual and auditory information and projects to the tectum, the red nucleus and the cerebral cortex via the thalamus. The cerebellum can be divided into three longitudinal zones (medial, intermediate and lateral) based on the projection of the cerebellar cortex onto the three deep nuclei. These zones differ in the type of information that comes into them via mossy fibers and climbing fibers, the two main inputs to the cerebellar cortex. The medial zone is dominated by information from vestibular, somatosensory, visual and auditory regions. The intermediate zone receives proprioceptive and somatosensory information from the spinal cord as well as information from the motor cortex via the ponto-cerebellar nuclei. The lateral zone receives information via the pontine nuclei from the motor cortex; the premotor and motor cortex (see Fig. 4). These differences in sensory input suggest functional differences. Indeed, experiments using rats with lesions targeting the different parts of the cerebellum revealed the functional specificity of the lateral part (comprising the cerebellar hemispheres and dentate nucleus) compared to the midline part (comprising the vermis and fastigial nucleus). Rats with midline lesions had disturbances in balance and equilibrium and presented visuo-motor functional deficits when assessed by the visible platform paradigm in the Morris water maze. Rats with lateral lesions had milder deficits and were deficient in spatial orientation (the 
hidden goal paradigm of the Morris water maze) but presented no visuo-motor deficit (visible platform paradigm) (Joyal et al., 1996). Lesions of the dentate nucleus alone did not affect equilibrium or balance performances but resulted in the same selective impairment in spatial orientation as that one observed with a combined lesion of the cerebellar hemispheres and dentate nucleus (Joyal et al., 2001). These results point towards a specific role of this lateral region in spatial orientation

Figure 4

\subsection{The cerebellum as a part of the spatial network}

The outputs of the cerebellum also strongly suggest a role in a network involved in spatial orientation. It has long been thought that outputs of the three zones of the cerebellar cortex (and therefore deep nuclei) were also segregated, i.e., fastigial to vestibular and reticulospinal systems, interpositus to red nucleus and dentate (via the thalamus) to motor cortex. Although there may be predominant segregation of the projections along these lines, there is also clear overlap (Bastian et al., 1999). Thus, vestibular, fastigial, interpositus and dentate nuclei all project via the thalamus to the motor cortex and vestibular, fastigial and interposed nuclei all project to the spinal cord (Asanuma et al., 1983a; Asanuma et al., 1983b). In primates (and probably in humans), the cerebellar projections of the lateral part (neo-cerebellum) also expand to almost all parts of the associative cortex including the frontal and parietal association areas (Glickstein, 1993). Nothing is known yet about such projections in rodents. In addition, electrophysiology recordings in rats suggest reciprocal projections between the cerebellum and the hippocampus. Both climbing and mossy fibers pathways, which are the two main inputs of the cerebellar cortex were activated by fornix 
stimulation (the main efferent pathway of the hippocampus) (Saint-Cyr and Woodward, 1980). Conversely, stimulation of the fastigial nucleus (corresponding to the lateral part) impaired neuronal responses in the septum and the hippocampus proper (Heath et al., 1978). It is not known whether these projections are direct or indirect.

2.3. The repetitive microcomplex and Long Term Depression cellular mechanism.

Ito was the first to propose the corticonuclear microcomplex as the functional module of the cerebellum (Ito, 2001). In this microcomplex, a cortical microzone is paired with a small distinct group of neurons in a deep cerebellar or vestibular nucleus. The mossy fibre (MF) afferents arising from various precerebellar nuclei supply excitatory synapses to granule cells in the microzone and also to the nuclear neurons via collaterals. Granule cell axons (called parallel fibers; PF) then send excitatory projections to the Purkinje cells. Another set of inputs to the microcomplex is relayed by a small distinct group of inferior olive neurons, whose axons end as climbing fibers (CF) on Purkinje cells and also supply excitatory synapses to the nuclear neurons via collaterals. According to Ito (2001), four basic notions constitute the operational principle of the microcomplex. First, MF signals to a microzone are relayed by granule cells and in turn excite Purkinje cells and other cortical neurons, eventually evoking simple spikes in Purkinje cells. Second, the MF signals drive the nuclear neurons, which generate output signals of the microcomplex under inhibitory influences of Purkinje cells. Third, CFs conveys error signals to a microcomplex regarding the operation of the neural system that includes the microcomplex. The error signals are generated by various 
neuronal mechanisms in diverse pre-olivary structures. Fourth, CF error signals induce the cerebellar synapse plasticity, Long Term Depression (LTD), in the conjointly activated PF-Purkinje cells synapses and thereby modify the operation of the microcomplex until the error signals are minimized.

LTD has been proposed to be a crucial cellular mechanism underlying motor learning (Marr, 1969; Albus, 1971; Ito and Kano, 1982). A large amount of experimental data supporting this theory are available in studies of simple forms of motor learning such as the vestibulo-ocular reflex (VOR) or eyelid conditioning (Gilbert and Thach, 1977; McCormick and Thompson, 1984). The crucial role of LTD during motor learning has been well demonstrated with recent work using a new transgenic model called L7-PKCI (De Zeeuw et al., 1998). This model has a specific alteration of LTD at the Purkinje cell level and a deficit of motor learning was observed during VOR adaptation (Goossens et al., 2001; Van Alphen and De Zeeuw, 2002). A question of interest is now to see whether this cellular synaptic mechanism could be involved in other kinds of learning such as spatial learning. Indeed, the diversity of the deficits described after cerebellar impairment could appear to be in contradiction with the repetitive anatomical structure defined as microcomplexe by Ito and found throughout the entire cerebellum. Whereas the potential role of the cerebellum in cognitive functions has been interpreted in many different ways, authors are currently trying to find a common process to all the deficits related to the cerebellum. The cerebellar LTD is one of the best cellular candidates.

2.4. The role of climbing fibers (CF) and parallel fibers (PF) 
The first indirect evidence for a potential role of cerebellar inputdependant mechanisms in the process of spatial navigation come from experimental lesions of these afferents. Our aim was to understand the specific and possible conjoint effect of a lesion of CF and PF inputs. Specific destruction of the inferior olivary neurons and their axons, the CFs, were made by i.p. administration of 3-acetylpyridine (3-AP) and niacinamide (Rondi-Reig et al., 2002). We used X-irradiation delivered to the cerebellum a few days after birth in order to prevent the genesis of granule cells and their axons, the PFs. The protocol of navigation we used was a "fixed departure-fixed arrival" procedure in the water maze. Animals had to find a hidden platform located in a fixed position. This protocol minimized spatial processing; to solve the task animals can use a single cue (rather than the configuration of cues in the room) or learn to orient their body directly to the platform. Our results demonstrated that the absence of the major cerebellar inputs, i.e. CF and PF, differentially impaired the acquisition of the non-visible platform task but not the visible platform task, suggesting a specific role of these inputs in spatial orientation rather than in visuo-motor orientation. A total lesion of PFs (with intact CFs) totally impaired the acquisition of the nonvisible version of the water maze (Le Marec et al., 1997). This impairment was the same in the case of a double lesion (PF and CF). In contrast to control animals that, after a few trials of random exploration, learned to use an initial rotation followed by a direct trajectory toward the platform, rats lacking PFs were unable to learn to orient their bodies toward the non-visible platform. Instead, they adopted a circling behavior during the majority of the trials. These data were comparable to those obtained by Petrosini et al (1996) in the "fixed start-fixed arrival" procedure (corresponding to the first protocol they used in their study). 
Hemicerebellectomized rats had trouble developing efficient searching behaviours: they performed extended searching around the pool and peripheral circling before finding the platform. These effects were present in the acquisition phase (i.e. when efficient exploration is extremely important). The similarity of the results strongly suggests that deficits observed after a lesion of the cerebellum could be due to its disconnection via one of its major inputs, the PFs. An additional experiment showed that partial destruction of the parallel fiber inputs only delayed the acquisition of the spatial task (Le Marec et al., 1997) therefore suggesting that the number of granule cells (and thus PFs) is a key factor in normal spatial function.

By contrast, a total lesion of CFs alone only delayed the acquisition of this same task (Dahhaoui et al., 1992). The delayed but not impaired behaviour observed with total CF destruction suggested a role of CF in the time course of the acquisition, its presence increasing the rapidity of the process. The importance of an intact functional CF system was additionally revealed in the case of a partial lesion of PFs only (Rondi-Reig et al., 2002). A total lesion of CFs in combination with a partial lesion of PFs dramatically impaired the acquisition of the nonvisible platform task (Rondi-Reig et al., 2002) whereas, as mentioned above, this acquisition was only delayed in the case of a partial lesion of PF and intact CF. All these results are summarized in Table 2.

Table 2

The comparison of the two types of lesions gave interesting insight into the relative importance of these two input systems. It seems that for this particular behavior, the presence of intact CFs can compensate for the absence of some of the PFs. It also suggests that the presence of PFs is crucial for the acquisition of 
such behavior, and that CFs play a role which seems minimal compared to that of the PFs. These results are consistent with the hypothesis of a possible role of a synaptic mechanism occurring at the level of the PFs. Indeed, the required presence of PFs for spatial learning and the importance of the presence of CFs when the number of PFs is decreased indicate a mechanism occurring at the PF level but also depending on CFs. Homo- and hetero-synaptic LTD occurring at the synaptic level of PF could be a good candidate.

Recent advances in conditional mutagenesis provide the required tool to address such a specific question. As mentioned above, L7-PKCI mice present a specific deficit of LTD at the synaptic level between PFs and Purkinje cells. In addition, this model presents no deficit in basic motor skills in contrast to all other cerebellar mutant models (Van Alphen and De Zeeuw, 2002). By testing these mutant mice for spatial navigation abilities, our first results revealed that they present spatial navigation deficit in the MWM (personal communication). We are currently characterizing these deficits in order to know if the procedural or declarative component is affected.

\section{Discussion}

Based on the accumulation of experimental data showing that cerebellar patients and animal models present cognitive deficits, what can one answer to the question: is the cerebellum ready for navigation? More than a decade after this question was first posed, one point is now recognized: the cerebellum must be added to the structural network involved in navigation. The anatomical projections 
of the cerebellum are consistent with this view. A crucial question nevertheless remains: how does the cerebellum participate? The role of the cerebellum in the procedural component of the navigation process is the aspect that has been the best documented experimentally. By contrast, the possible implication of the cerebellum in the declarative component of navigation remains unclear. Is the information processing in the cerebellum required for the acquisition of a spatial representation? Is the procedural impairment observed in cerebellar animals indirectly responsible for an impaired spatial representation? The possible bidirectional projections between the cerebellum and the hippocampus revealed by electric stimulation are intriguing. However, anatomical analyses to confirm such projections are lacking.

A computational model has suggested a complementary role of the cerebellum and the basal ganglia for the learning of sequential procedures (Hikosaka et al., 1999). Such a model is consistent with the parallel anatomical loops formed with cortical area, in particular the prefrontal cortex (Middleton and Strick, 2000; Middleton and Strick, 2001), by the lateral part of the cerebellum on the one hand and the caudate nucleus-substantia nigra pathway on the other (see Fig. 5).

Figure 5

Recent studies have shown that brain areas other than the cerebellum contribute to different stages and aspects of procedural learning (Doya, 2000). On the basis of a series of studies using a sequence-learning task with trial-and-error in monkeys, Hikosaka and collaborators (Hikosaka et al., 1999) have proposed a hypothetical scheme in which a sequential procedure is acquired independently by two cortical systems, one based on basal ganglia and using spatial coordinates and 
the other based on the cerebellum using motor coordinates. These systems would be preferentially active in the early (basal ganglia) and late (cerebellum) stages of learning. Both of the two systems are supported by loop circuits formed with the basal ganglia and the cerebellum, the former for reward-based evaluation and the latter for processing of timing.

Another unanswered question concerns the potential mechanism(s) underlying the function of the cerebellum during navigation. As mentioned above, a number of apparently different functions have been attributed to the cerebellum, from basic motor skills to cognitive function, yet the internal anatomy of the cerebellum is highly homogenous. One possibility would be to consider that all these functions have a common process in which the cerebellum participates. This proposition is also consistent with a recent finding showing that protein kinase Cdependent long-term depression in Purkinje cells is necessary for learningdependent timing (Koekkoek et al., 2003) as proposed in the model of Hikosaka et al. (1999). We are currently trying to determine whether this process is also necessary for spatial learning.

\section{Acknowledgements}

The authors would like to thank Anne Lohof for reviewing the english. Work in the author's laboratory is supported by grants from the ACI "Neurosciences Intégratives et Computationnelles” (NIC 0083) and the European Laboratory of Neurosciences and Action (LENA). 
References

Albus, J. (1971) A theory of cerebellar function. Math Biosci 10, 26-61.

Asanuma, C., Thach, W. T., and Jones, E. G. (1983a) Brainstem and spinal projections of the deep cerebellar nuclei in the monkey, with observations on the brainstem projections of the dorsal column nuclei. Brain Res 286, 299-322.

Asanuma, C., Thach, W. T., and Jones, E. G. (1983b) Distribution of cerebellar terminations and their relation to other afferent terminations in the ventral lateral thalamic region of the monkey. Brain Res 286, 237-265.

Bastian, AJ., Mugnaini, E., and Thach, WT. (1999) Cerebellum. In: Fundamental Neuroscience, pp. 973-992. Eds Zigmund MJ, Bloom FE, Landis SC, Roberts JL, Squire IR.

Botez, M. I., Botez, T., Elie, R., and Attig, E. (1989) Role of the cerebellum in complex human behavior. Ital J Neurol Sci 10, 291-300.

Botez-Marquard, T. and Botez, M. I. (1992) Visual memory deficits after damage to the anterior commissure and right fornix. Arch Neurol 49, 321-324.

Dahhaoui, M., Stelz, T., and Caston, J. (1992) Effects of lesion of the inferior olivary complex by 3-acetylpyridine on learning and memory in the rat. J Comp Physiol [A] 171, 657-664.

De Zeeuw, C. I., Hansel, C., Bian, F., Koekkoek, S. K., van Alphen, A. M., Linden, D. J., and Oberdick, J. (1998) Expression of a protein kinase C inhibitor in Purkinje cells blocks cerebellar LTD and adaptation of the vestibulo-ocular reflex. Neuron 20, 495-508.

Doya, K. (2000) Complementary roles of basal ganglia and cerebellum in learning and motor control. Curr Opin Neurobiol 10, 732-739.

Drepper, J., Timmann, D., Kolb, F. P., and Diener, H. C. (1999) Non-motor associative learning in patients with isolated degenerative cerebellar disease. Brain 122 ( Pt 1), 87-97.

Fortier, P. A., Smith, A. M., and Rossignol, S. (1987) Locomotor deficits in the mutant mouse, Lurcher. Exp Brain Res 66, 271-286.

Gandhi, C. C., Kelly1 RM, Wiley, R. G., and Walsh, T. J. (2000) Impaired acquisition of a Morris water maze task following selective destruction of cerebellar purkinje cells with OX7saporin. Behav Brain Res 109, 37-47.

Gilbert, P. F. and Thach, W. T. (1977) Purkinje cell activity during motor learning. Brain Res 128, 309-328.

Glickstein, M. (1993) Motor skills but not cognitive tasks. Trends Neurosci 16, 450-451.

Gomez-Beldarrain, M., Garcia-Monco, J. C., Rubio, B., and Pascual-Leone, A. (1998) Effect of focal cerebellar lesions on procedural learning in the serial reaction time task. Exp Brain Res 120, 25-30. 
Goodlett, C. R., Hamre, K. M., and West, J. R. (1992) Dissociation of spatial navigation and visual guidance performance in Purkinje cell degeneration (pcd) mutant mice. Behav Brain Res 47, 129-141.

Goossens, J., Daniel, H., Rancillac, A., van der, S. J., Oberdick, J., Crepel, F., De Zeeuw, C. I., and Frens, M. A. (2001) Expression of protein kinase C inhibitor blocks cerebellar longterm depression without affecting Purkinje cell excitability in alert mice. J Neurosci 21, 58135823.

Grafman, J., Litvan, I., Massaquoi, S., Stewart, M., Sirigu, A., and Hallett, M. (1992) Cognitive planning deficit in patients with cerebellar atrophy. Neurology 42, 1493-1496.

Heath, R. G., Dempesy, C. W., Fontana, C. J., and Myers, W. A. (1978) Cerebellar stimulation: effects on septal region, hippocampus, and amygdala of cats and rats. Biol Psychiatry 13, 501-529.

Hikosaka, O., Nakahara, H., Rand, M. K., Sakai, K., Lu, X., Nakamura, K., Miyachi, S., and Doya, K. (1999) Parallel neural networks for learning sequential procedures. Trends Neurosci 22, 464-471.

Hilber, P., Jouen, F., Delhaye-Bouchaud, N., Mariani, J., and Caston, J. (1998) Differential roles of cerebellar cortex and deep cerebellar nuclei in learning and retention of a spatial task: studies in intact and cerebellectomized lurcher mutant mice. Behav Genet 28, 299-308.

Holmes G. (1939) The cerebellum of man. Brain 62, 1-30.

Hubrich-Ungureanu, P., Kaemmerer, N., Henn, F. A., and Braus, D. F. (2002) Lateralized organization of the cerebellum in a silent verbal fluency task: a functional magnetic resonance imaging study in healthy volunteers. Neurosci Lett 319, 91-94.

Ito, M. (2001) Cerebellar long-term depression: characterization, signal transduction, and functional roles. Physiol Rev 81, 1143-1195.

Ito, M. and Kano, M. (1982) Long-lasting depression of parallel fiber-Purkinje cell transmission induced by conjunctive stimulation of parallel fibers and climbing fibers in the cerebellar cortex. Neurosci Lett 33, 253-258.

Ivry, R. (1997) Cerebellar timing systems. Int Rev Neurobiol 41, 555-573.

Joyal, C. C., Meyer, C., Jacquart, G., Mahler, P., Caston, J., and Lalonde, R. (1996) Effects of midline and lateral cerebellar lesions on motor coordination and spatial orientation. Brain Res 739, 1-11.

Joyal, C. C., Strazielle, C., and Lalonde, R. (2001) Effects of dentate nucleus lesions on spatial and postural sensorimotor learning in rats. Behav Brain Res 122, 131-137.

Koekkoek, S. K., Hulscher, H. C., Dortland, B. R., Hensbroek, R. A., Elgersma, Y., Ruigrok, T. J., and De Zeeuw, C. I. (2003) Cerebellar LTD and learning-dependent timing of conditioned eyelid responses. Science 301, 1736-1739.

Lalonde, R. (1997) Visuospatial abilities. Int Rev Neurobiol 41, 191-215. 
Lalonde, R. and Thifault, S. (1994) Absence of an association between motor coordination and spatial orientation in lurcher mutant mice. Behav Genet 24, 497-501.

Le Marec, N., Dahhaoui, M., Stelz, T., Bakalian, A., Delhaye-Bouchaud, N., Caston, J., and Mariani, J. (1997) Effect of cerebellar granule cell depletion on spatial learning and memory and in an avoidance conditioning task: studies in postnatally X-irradiated rats. Brain Res Dev Brain Res 99, 20-28.

Leggio, M. G., Neri, P., Graziano, A., Mandolesi, L., Molinari, M., and Petrosini, L. (1999) Cerebellar contribution to spatial event processing: characterization of procedural learning. Exp Brain Res 127, 1-11.

Leiner, H. C., Leiner, A. L., and Dow, R. S. (1993) Cognitive and language functions of the human cerebellum. Trends Neurosci 16, 444-447.

Mandolesi, L., Leggio, M. G., Graziano, A., Neri, P., and Petrosini, L. (2001) Cerebellar contribution to spatial event processing: involvement in procedural and working memory components. Eur J Neurosci 14, 2011-2022.

Mandolesi, L., Leggio, M. G., Spirito, F., and Petrosini, L. (2003) Cerebellar contribution to spatial event processing: do spatial procedures contribute to formation of spatial declarative knowledge? Eur J Neurosci 18, 2618-2626.

Marr, D. (1969) A theory of cerebellar cortex. J Physiol 202, 437-470.

Martin, L. A., Goldowitz, D., and Mittleman, G. (2003) The cerebellum and spatial ability: dissection of motor and cognitive components with a mouse model system. Eur J Neurosci 18, 2002-2010.

McCormick, D. A. and Thompson, R. F. (1984) Neuronal responses of the rabbit cerebellum during acquisition and performance of a classically conditioned nictitating membrane-eyelid response. J Neurosci 4, 2811-2822.

Middleton, F. A. and Strick, P. L. (2000) Basal ganglia and cerebellar loops: motor and cognitive circuits. Brain Res Brain Res Rev 31, 236-250.

Middleton, F. A. and Strick, P. L. (2001) Cerebellar projections to the prefrontal cortex of the primate. J Neurosci 21, 700-712.

Molinari, M., Grammaldo, L. G., and Petrosini, L. (1997) Cerebellar contribution to spatial event processing: right/left discrimination abilities in rats. Eur J Neurosci 9, 1986-1992.

Morris, R., Rawlins, J., Garrud, P., and O'Keefe, J. (1984) Place navigation impaired in rats with hippocampal lesions. Nature 297(5868), 681-683.

Petrosini, L., Leggio, M. G., and Molinari, M. (1998) The cerebellum in the spatial problem solving: a co-star or a guest star? Prog Neurobiol 56, 191-210.

Petrosini, L., Molinari, M., and Dell'Anna, M. E. (1996) Cerebellar contribution to spatial event processing: Morris water maze and T-maze. Eur J Neurosci 8, 1882-1896. 
Rondi-Reig, L., Le Marec, N., Caston, J., and Mariani, J. (2002) The role of climbing and parallel fibers inputs to cerebellar cortex in navigation. Behav Brain Res 132, 11-18.

Saint-Cyr, J. A. and Woodward, D. J. (1980) Activation of mossy and climbing fiber pathways to the cerebellar cortex by stimulation of the fornix in the rat. Exp Brain Res 40, 112.

Schenk, F. and Morris, R. G. (1985) Dissociation between components of spatial memory in rats after recovery from the effects of retrohippocampal lesions. Exp Brain Res 58, 11-28.

Schmahmann, J. D. and Sherman, J. C. (1997) Cerebellar cognitive affective syndrome. Int Rev Neurobiol 41, 433-440.

Thach, W. T. (1998) A role for the cerebellum in learning movement coordination. Neurobiol Learn Mem 70, 177-188.

Thompson, R. F. and Kim, J. J. (1996) Memory systems in the brain and localization of a memory. Proc Natl Acad Sci U S A 93, 13438-13444.

Van Alphen, A. M. and De Zeeuw, C. I. (2002) Cerebellar LTD facilitates but is not essential for long-term adaptation of the vestibulo-ocular reflex. Eur J Neurosci 16, 486-490. 
List of abbreviations

CF: climbing fibers

LTD: Long-term depression

MF: Mossy fibers

MWM: Morris water maze

PF: Parallel fibers

VOR: Vestibulo-ocular reflex 
Figure Legends

Figure 1

The Morris water maze paradigm. (A) During the non-visible platform task, animals are required to find an immerged and unvisible platform (see dashed circle) located in a fixed position from random-departure points (indicated by stars). In order to solve this task, animals can use extra-maze cues located in the environment. (B) During the visible platform task the platform was submerged and visible. A proximal cue (represented by flags) is placed on the top of the submerged platform, therefore indicated the exact position of the platform to the animal. The visible platform is positioned in one of four possible locations (north, east, west, south) as indicated on the figure.

Figure 2

The Rey Complex Figure Test is a widely used neuropsychological test for visuoconstructive skills and visual memory. This drawing and visual memory test examines the ability to construct a complex figure and remember it for later recall. The patient's methods of procedure as well as his specific copying errors are analyzed. It measures memory as well as visual-motor organization.

\section{Figure 3}

To establish whether a cerebellar lesion would cause a deficit in spatial navigation, hemi-cerebellectomized rats (Hcbed) were tested in an MWM paradigm characterized by a first phase with the platform hidden in a pool position (place I phase), followed by a phase with a visible platform moved in a different pool 
position (cue phase), followed by a final phase with the platform hidden in the last position (place II phase). Mean escape latencies in MWM is represented. Rats were pre-operatively (place I, cue and place II) and post-operatively (place III) tested. Note the maintenance of the direct trajectory acquired preoperatively. Vertical arrows indicate hemicerebellectomy (HCb). Vertical bars indicate standard errors. [from Petrosini et al. (1996)].

Figure 4

Anatomical projections from and to the cerebellum. The cerebellum receives multisensorial inputs via pre-cerebellar nuclei. The different parts of the cerebellar cortex projects to three cerebellar nuclei and are connected to the cortical area via the thalamus and to sensori-motor related structures via the red nucleus.

\section{Figure 5}

Parallel anatomical loops of the lateral part of the cerebellum and of the basal ganglia with the prefrontal cortex. 
Figure 1

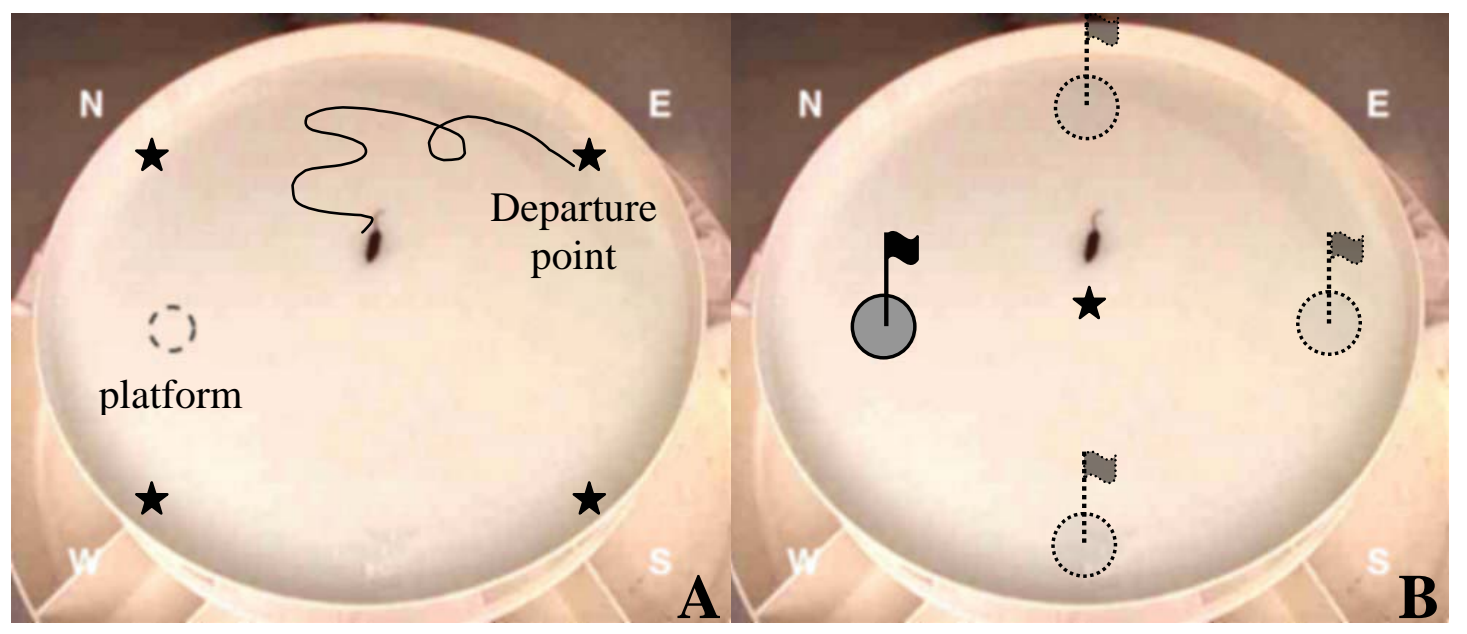


Figure 2
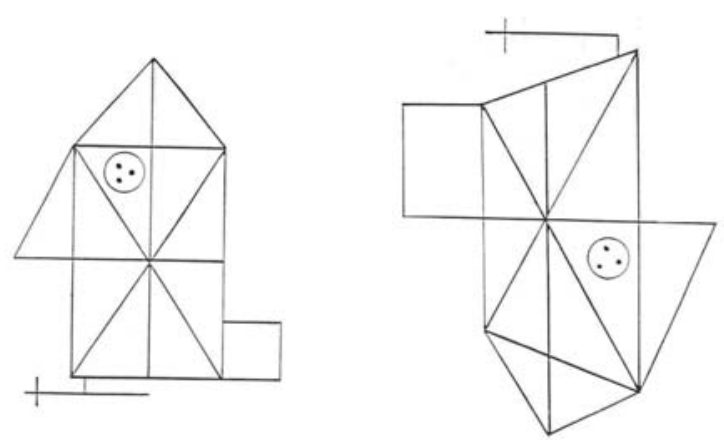
Figure 3

\section{$\mathrm{HCb}$}
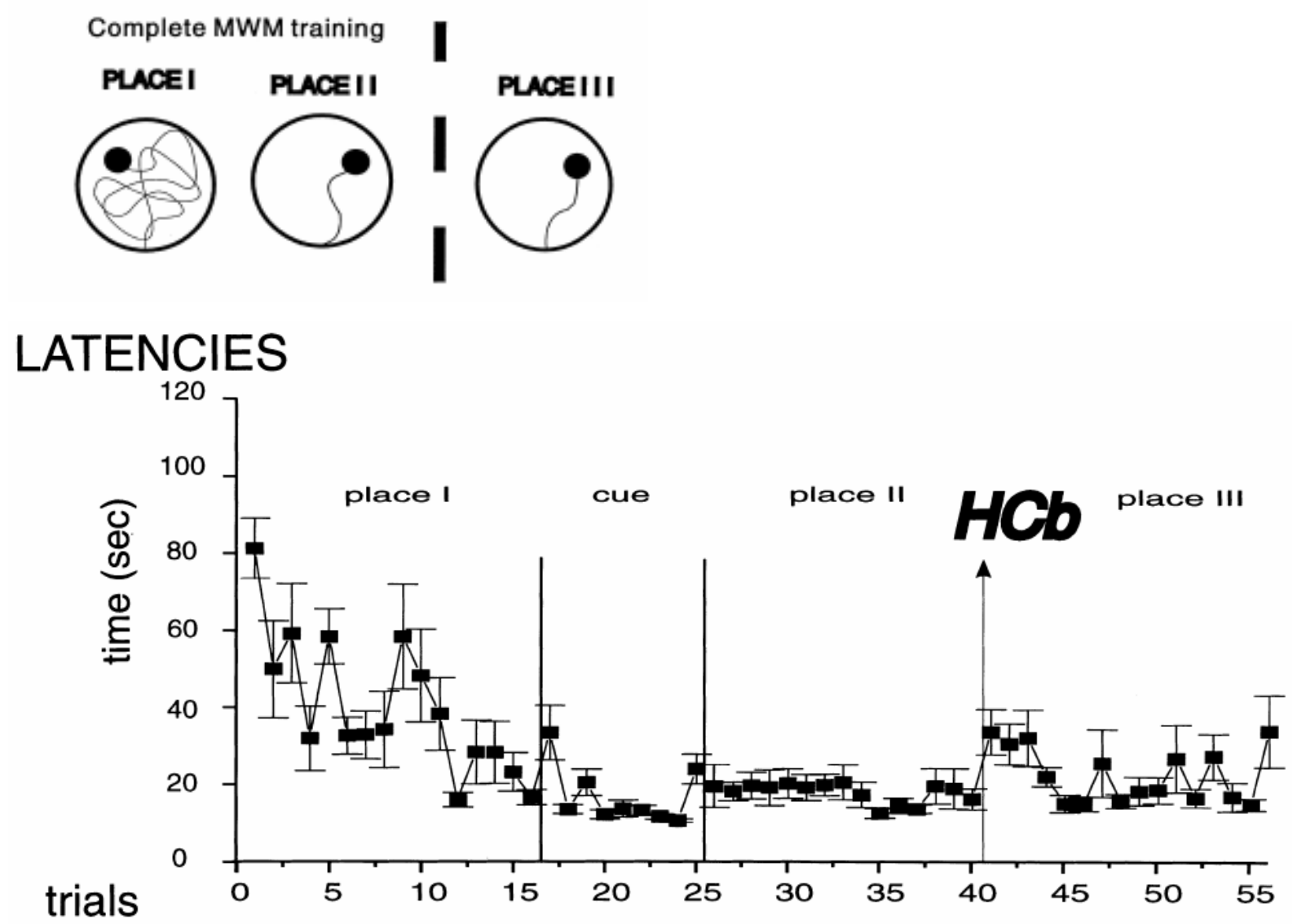

From Petrosini et al., 1996 


\section{Figure 4}

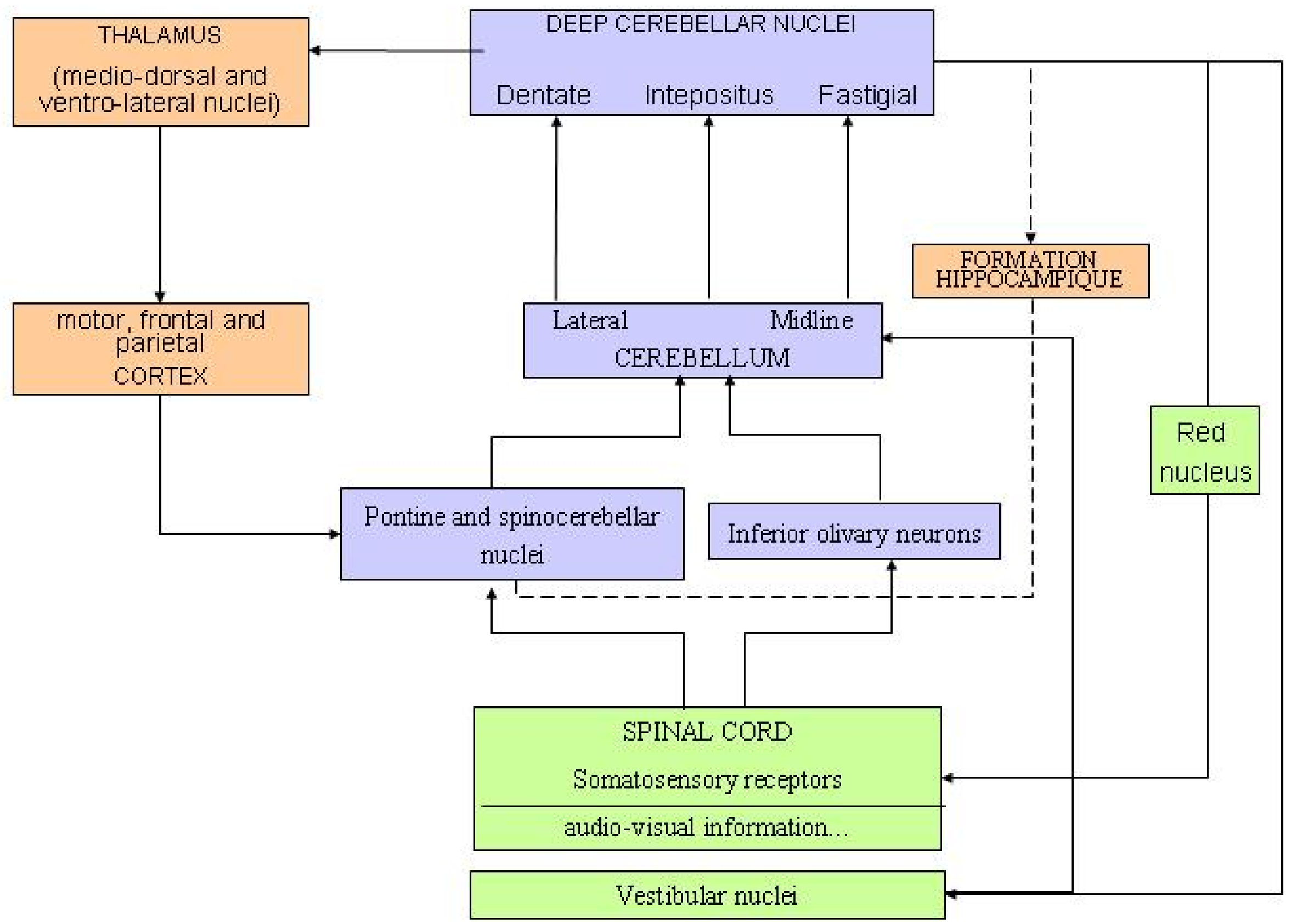


Figure 5

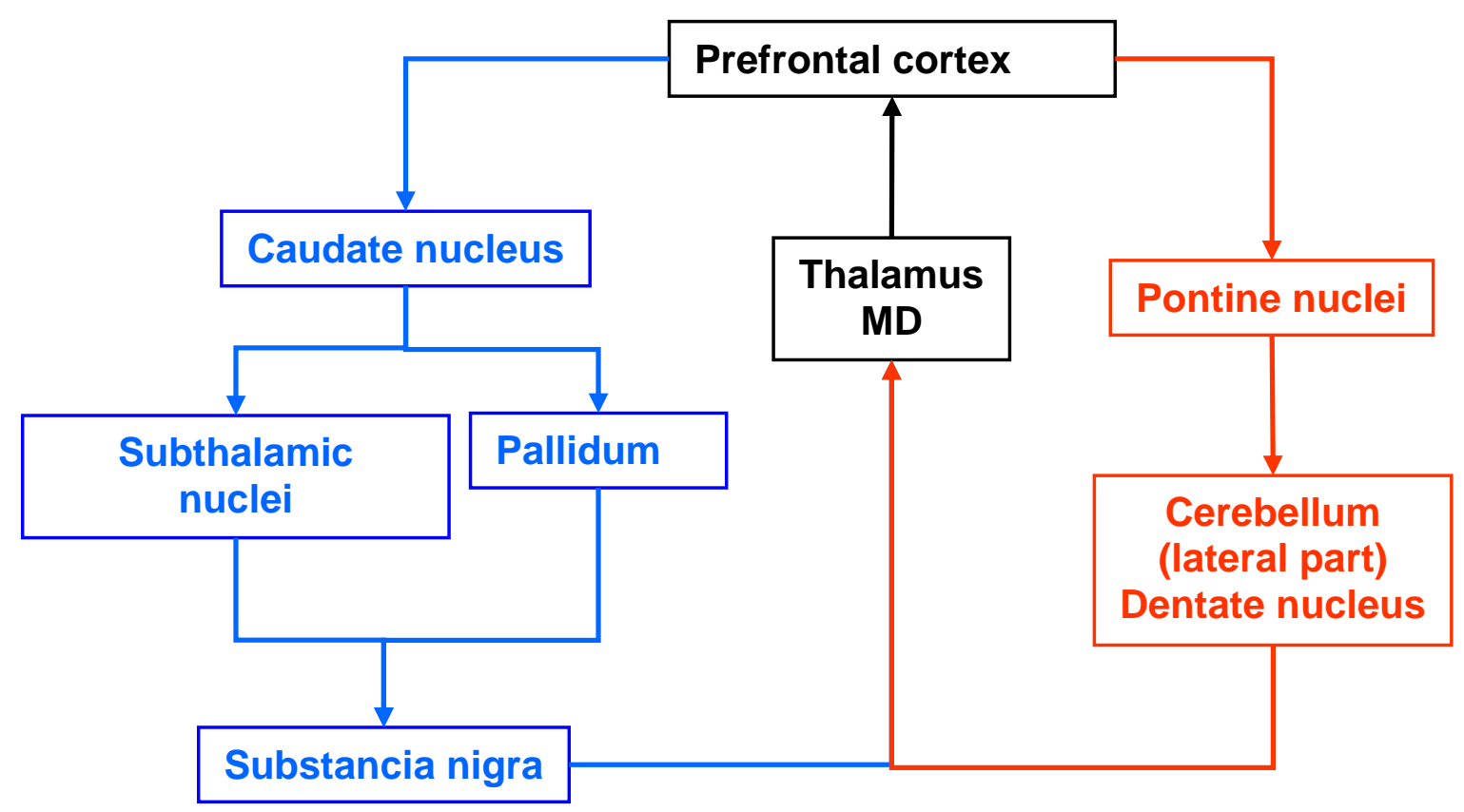


Table 1

Spatial impairments in cerebellar animals models

\begin{tabular}{|l|l|l|l|l|l|l|}
\hline & \multicolumn{1}{|c|}{$\begin{array}{c}\text { Type of } \\
\text { impairment }\end{array}$} & $\begin{array}{c}\text { Water maze: } \\
\text { hidden } \\
\text { platform }\end{array}$ & $\begin{array}{l}\text { Visible } \\
\text { platform }\end{array}$ & $\begin{array}{c}\text { Motor } \\
\text { deficit }\end{array}$ & $\begin{array}{c}\text { Cerebellar } \\
\text { Cortex } \\
\text { impairment }\end{array}$ & $\begin{array}{c}\text { Extracerebellar } \\
\text { cortex } \\
\text { impairment }\end{array}$ \\
\hline Lurcher & mutant & Yes & Yes & Yes & $\begin{array}{l}\text { Granule, } \\
\text { Purkinje }\end{array}$ & Inferior olive \\
\hline Staggerer & mutant & Yes & Yes & Yes & $\begin{array}{l}\text { Granule, } \\
\text { Purkinje }\end{array}$ & Inferior olive \\
\hline Weaver & mutant & Yes & Yes & Yes & Granule & $\begin{array}{l}\text { Substantia } \\
\text { Nigra } \\
\text { pars compacta }\end{array}$ \\
\hline pcd & mutant & Yes & No & Yes & Purkinje & Inferior olive \\
\hline nervous & mutant & Yes & No & Yes & Purkinje & $\begin{array}{l}\text { Deep cerebellar } \\
\text { nuclei }\end{array}$ \\
\hline OX7 & Immunotoxin & Yes & No & $\begin{array}{l}\text { No } \\
\text { Locomotor } \\
\text { activity }\end{array}$ & Purkinje & No \\
\hline
\end{tabular}

Weaver : in this case, the paradigm was an aquatic maze with walls. The animals presented an increased number of errors compared to controls and never used a direct path to the goal.

Adapted from Lalonde et al., 1999 
Table 2

Functional consequences of climbing fibres and parallel fibers lesions

\begin{tabular}{|l|c|c|}
\hline & Non-visible task & Visible task (cued) \\
\hline 1. Controls & normal & normal \\
\hline 2. Total lesion of CF/Intact PF & delayed & (not done) \\
\hline 3. Intact CF/Partial lesion of PF & delayed & (not done) \\
\hline $\begin{array}{l}\text { 4. Intact CF/Total lesion of PF } \\
\text { (partially lesioned group) }\end{array}$ & impaired & normal \\
\hline $\begin{array}{l}\text { 6. Total lesion of CF/Partial lesion of PF } \\
\text { (totally lesioned group) }\end{array}$ & impaired & \\
\hline
\end{tabular}

Delayed means that experimental animals learn the task and finally reach similar escape latencies than controls but need more training trials. Impaired means that experimental animals never learn the task and present significantly higher escape latencies compared to controls even at the end of the training.

From Rondi-Reig et al., 2002 